\title{
Breast arterial calcifications as an indicator of atherosclerotic cardiovascular disease: comparative analysis of coronary computed tomography scoring systems and carotid intima-media thickness
}

\author{
Aykut Kadıŏlu^, Suzan Bahadır \\ Radiology Department, Başkent University Alanya Medical Research and Training Hospital, Antalya, Turkey \\ Contributions: (I) Conception and design: A Kadığlu; (II) Administrative support: A Kadığlu; (III) Provision of study materials or patients: A \\ Kadığlu; (IV) Collection and assembly of data: A Kadığlu; (V) Data analysis and interpretation: Both authors; (VI) Manuscript writing: Both \\ authors; (VII) Final approval of manuscript: Both authors.
}

Correspondence to: Aykut Kadığlu. Radiology Department, Başkent University Alanya Medical Research and Training Hospital, Antalya, Turkey. Email: aykutkad@gmail.com.

Background: Breast arterial calcification (BAC) is easily detected and commonly observed on screening
mammography. That is more frequent among people with diabetes, and these people are at risk of coronary
artery disease. The incidence of BAC increases with advancing age. We aimed to determine whether BAC
detected by mammography is associated with the development of coronary atherosclerosis in asymptomatic
women. It can help reduce morbidity and mortality secondary to atherosclerotic cardiovascular disease.
Methods: We included one hundred and eighty women over the age of 40 who underwent mammography
screening in this multi-modality study. Mammography evaluated the presence of calcifications, the number
of involved arteries, and the distribution. We questioned the patients about cardiovascular risk factors such
as hypertension and diabetes. The coronary artery disease severity was assessed according to both Agatston
and calcium scores on coronary computed tomography (CT). Besides, the relationship between these scores
and correlation with carotid artery intima-media thickness was investigated. We stated mean and standard
deviation (SD) for continuous variables and reported frequency distributions and percentages. SPSS software
version 25.0 was used to perform the analysis. Results: Overall, 302 of 3,600 cases were positive for BAC. However, 120 of them could be included in the study by the eligibility criteria of our research. In univariate analysis, age, hyperlipidemia, DM, HT, and smoking history were risk factors that significantly affected BAC development. The impact of age and diabetes were maintained in the logistic regression analysis $(\mathrm{P}<0.005)$, while the significant effect of the other variables was vanished $(\mathrm{P}>0.02)$. Furthermore, moderate and high BAC scores were correlated with higher coronary atherosclerosis scores.

Conclusions: BAC may predict an additional risk factor for coronary artery disease, particularly in patients having higher scores. That may be an accurate indicator for subsequent development of coronary arterial calcifications so that it may be possible to reduce morbidity and mortality associated with coronary atherosclerosis.

Keywords: Breast; atherosclerosis; coronary artery disease (CAD); mammography; computed tomography angiography

\footnotetext{
$\wedge$ ORCID: 0000-0001-8955-9449.
} 
Submitted Jan 26, 2021. Accepted for publication May 27, 2021.

doi: $10.21037 /$ qims-21-98

View this article at: https://dx.doi.org/10.21037/qims-21-98

\section{Introduction}

Mammography is an established, widely accepted, and easily accessible imaging modality to diagnose breast cancer in the early phase. The women undergoing mammography screening are candidates for atherosclerotic cardiovascular disease (ASCVD) because of age $(1,2)$. However, the clinical importance of the data representing subclinical atherosclerosis is frequently ignored during the routine assessment. This data includes breast arterial calcification (BAC), which is related to an increased risk of cardiovascular disease (CVD). BAC is a benign and incidental finding from an oncological perspective, and mammographic detection can predict whether a patient has a high risk for CAD. Such knowledge may be helpful in the improvement of CVD risk analysis like coronary arterial calcification (CAC) has been verified by numerous studies $(3,4)$. Vascular calcification is a significant and independent risk factor for mortality and morbidity. Therewithal, BAC has been identified as a "women-specific risk marker" for ASCVD. For a long time, vascular calcification is believed to be a passive part of aging, and "wear and tear" are now considered an active, cell-mediated complex process that is regulated but not yet fully elucidated (5-7).

BAC is encountered as an incidental finding which is uncommon in women younger than 50 years of age, emerges most likely by increasing age. There is a characteristic pattern of linear amorphous calcification bounded by two parallel lines forming a tram-track appearance. That involves the entire circumference of the vessel and precisely represents arteriosclerosis of peripheral arteries. ASCVD is one of the most important causes of morbidity and mortality in older women (6-9). That is defined as a multifactorial chronic inflammatory disease with a heterogeneous clinical picture. Age, diabetes mellitus (DM), hypertension (HT), smoking, and hyperlipidemia are shown as independent risk factors. Quantitative measurements such as carotid intima-media thickness (C-IMT) and coronary computed tomography (CT) calcium score, considered a valuable indicator for atherosclerosis, are used in radiological practice. It is predicted that BAC detected in mammography screening can be used as an indicator of CAD. However, in some coronary artery studies, it is claimed that BAC may not be an accurate marker for coronary atherosclerosis (10-15).

This multi-modality study aimed to examine the relationship between BAC detected by mammography screening and some other risk factors quantitatively, and thus to evaluate whether BAC could be a "female-specific indicator" of coronary atherosclerosis.

\section{Methods}

This retrospective study was conducted in accordance with the Declaration of Helsinki (as revised in 2013). The ethics committee of Başkent University Faculty of Medicine approved the study, and informed consent was taken from all the patients. Breast imaging services include digital mammography, breast ultrasound, and breast magnetic resonance imaging (MRI) if needed as a further imaging modality at our medical research and training center. A physician referral or order is usually required. Patients were questioned about smoking, cardiac disease, HT, DM, hyperlipidemia by the patient data form as a routine procedure and recorded data for each patient. The responses were entered into the hospital data system. HT was defined as diastolic blood pressure above $90 \mathrm{mmHg}$, or systolic blood pressure is above $140 \mathrm{mmHg}$ or antihypertensive medication. DM was diagnosed as follows; if the fasting plasma glucose concentration was higher than $126 \mathrm{mg} / \mathrm{dL}$ on two different occasions, or if the patient was on treatment with an antidiabetic agent. A patient having a total cholesterol level above $200 \mathrm{mg} / \mathrm{dL}$ was identified as hyperlipidemia. Smoking was described as current smoking or having a history of smoking habits. An active smoker was described as a respondent who had smoked at least half a pack a day for more than 5 years. We aimed to reach a statistically more significant and reliable result about smoking, BAC, and coronary atherosclerosis association by determining such a smoking intensity model.

We included the patients who underwent mammography examination between the 1st of January 2015 and the 1st of December 2020 in this study. Overall, 3,600 mammography screenings were evaluated, and 302 were identified as BAC (+) patients. Likewise, by retrospective review of the imaging database at the medical research and training center, images of all patients undergoing both mammography screening 


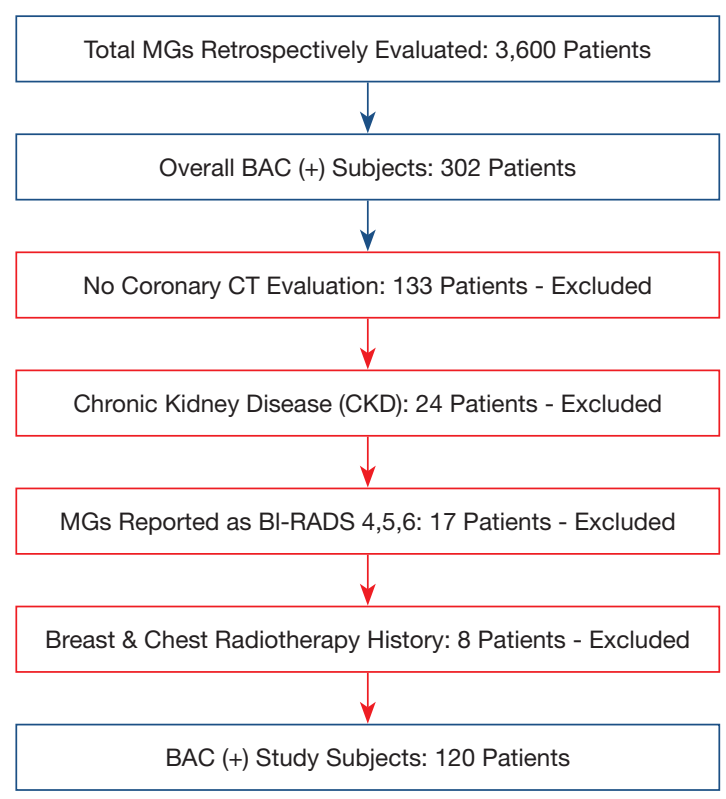

Figure 1 Formation scheme of BAC (+) study group. Sixty BAC (-) subjects were included as the control group to make a comparative statistical analysis. BAC, breast arterial calcification; MG, mammography; BI-RADS, Breast Imaging-Reporting and Data System.

and coronary CT (calcium scoring) within 5 years were analyzed. Images of the patients who underwent coronary CT (angiography and calcium scoring), carotid artery Doppler US examination, and mammography within 1 year were analyzed. We excluded 17 cases having suspicious malignant findings or histopathologically proven malignant lesions on mammography, which had been reported as Breast Imaging-Reporting and Data System (BI-RADS) 4, 5 , or 6 . The number of patients excluded from the study because of breast or chest region radiotherapy history was 8 ; radiotherapy may be the reason for dystrophic calcifications, lipoid necrosis. Besides, 24 patients having chronic kidney disease (CKD) were not included in the study because CKD is a proven and well-known vital risk factor of atherosclerosis, vascular calcifications $(9,14-17)$. We had to exclude 133 subjects who had no coronary CT evaluation in our digital archives. Among the remaining individuals, 120 BAC (+) females were identified, determination of the case group and age distribution were shown in Figure 1 and Table 1, respectively. Also, 60 BAC (-) mammography screenings were included in the study as the control group. BAC (-) patients were randomly selected, and this group was formed by a similar age distribution with
Table 1 Distribution of BAC (+) and BAC (-) patients by age, 10 -year pooled $(\mathrm{n}=180)$

\begin{tabular}{lcc}
\hline Age (years) & BAC $(+), \mathrm{n}(\%)$ & BAC $(-), \mathrm{n}(\%)$ \\
\hline $40-49$ & $46(38.3)$ & $24(40.0)$ \\
$50-59$ & $35(29.2)$ & $16(26.7)$ \\
$60-69$ & $22(18.3)$ & $11(18.3)$ \\
$70-79$ & $12(10.0)$ & $6(10.0)$ \\
$\geq 80$ & $5(4.2)$ & $3(5.0)$ \\
Total & $120(100.0)$ & $60(100.0)$ \\
\hline
\end{tabular}

BAC, breast arterial calcification.

BAC (+) group, regardless of chronic disease history. Ultrasonographic C-IMT measurements in all of these 180 participants were recorded for each from the digital database. CAC scores, C-IMT values were all compared so that the presence of statistically significant difference was investigated. Moreover, whether chronic diseases have a substantial effect on BAC was evaluated statistically.

One hundred and eighty patients were included in the study, as shown in Table 1. The median age of all BAC $(+)$ and BAC (-) groups was approximately 56 years. Respondents' ages were between 40 and 86 in BAC (+) group and between 40 and 83 in BAC (-) group. All patients in the study were of the Turkish race.

Standard digital mammograms (full-field) were acquired in the craniocaudal (CC) and mediolateral oblique (MLO) positions on a GE Senographe 800T, General Electric (Buc-Cedex, France). All mammograms were assessed on standard 5.1-megapixel mammography monitors by two radiologists who were experienced more than 7 years and were blinded to each other's BAC evaluation reports, patients' clinical history, and coronary calcium score.

The coronary calcium score is calculated by multiplying the area of calcification by the weighted value assigned to its highest Hounsfield unit (HU) and summed for all lesions on CT. This quantification is defined as the Agatston score. The calculated scores were classified as follows in leveling the risk for $\mathrm{CAD}$ (coronary artery disease); 0 no risk, $1-10$ minimal, 11-100 mild, 101-400 moderate; $>400$ high risks. The percentile distribution of calcium score adjusted by age, gender, and the race was calculated based on the MultiEthnic Study of Atherosclerosis (MESA). MESA excluded patients having diabetes and chronic renal disease to reduce the effect of main secondary factors (10). In this study, we made statistical evaluations by measuring the coronary 
calcium score with both the Agatston $(0-1,000)$ system and the CAC score (0-12) as previously performed by Shemesh et al. (18). According to the second one; each of the four main coronary arteries was identified (left main, left anterior descending, circumflex, and right), and the existence of CAC in each artery was categorized as absent, mild, moderate, or severe and scored as $0,1,2$, or 3, respectively. The CAC was classified as mild when less than one-third of the length of the entire artery showed calcification, moderate when one-third to two-thirds of the artery showed calcification, and severe when more than two-thirds of the artery showed calcification. With four arteries thus scored, each participant received a score from 0 to 12 . The CAC scores were divided into three categories of increasing severity: 0,1 to 3 , and 4 to 12 , which are firmly predictive of cardiac outcomes in several studies $(19,20)$. CT evaluations were acquired by Siemens SOMATOM Sensation 64 eco CT scanner (Wuxi, Jiangsu, China). We included both Agatston and CAC scores in the study because we use these scores in routine radiological practice. Therefore, we aimed to reinforce the statistical value of the results obtained by conducting a detailed comparative analysis.

Furthermore, ultrasonographic evaluation and measurement of C-IMT were made by the single radiologist who was blinded to mammography findings and the clinical data, and all patients were examined by using the Siemens Acuson S2000 Ultrasound system (Mountain View, CA94043, USA). C-IMT measurements were made with the $\mathrm{B}$-mode ultrasound examination by using a $12-\mathrm{MHz}$ linear array transducer. The common carotid artery (CCA) was examined on both sides; meanwhile, the respondent was lying supine. The head was directed away from the side of interest, and the neck was slightly extended position. The distal segment of CCA that is $1 \mathrm{~cm}$ proximal to the carotid bifurcation was identified. The C-IMT of the distal wall was evaluated as the distance between the lumen-intima interface and the media-adventitia interface. Bilateral C-IMT measurements were obtained from three adjacent segments at 1-2 $\mathrm{mm}$ intervals, and the average of these three was used for analyses. The average of both carotids was recorded as the C-IMT value. While measuring IMT, the carotid plaques were not considered.

As applied previously in several studies, the scoring system was used to make a robust comparative quantitative analysis of the severity of atherosclerosis in women with BAC $(11,12)$. The scoring system was as follows; the number of vessels involved in each breast was recorded and numerically coded as 1 to 6 ; if there were more than six calcified vessels, it was coded as 6 . According to the length of vascular segment involvement, it was coded as follows; none (scored as 0), less than one-third (scored as 1), between one-third and two-thirds (scored as 2), and greater than two-thirds (scored as 3). The calcium density of the most severely affected vascular segment was recorded as follows; none (scored as 0 ), mild with clear visualization of the lumen or only one vessel wall involved (scored as 1), moderate with clouding of the lumen, and calcification of both tangential walls (scored as 2), and severe with no visible lumen (scored as 3). Each participant has received a BAC score between 0 and 12 after summing up all these three numbers for each breast. If scores were different for each breast in a patient, then the highest value was recorded as the one final score for each case. The BAC results were also classified into three categories according to their severity; 0,1 to 3 , and 4 to 12 . The frequency of calcified breast arteries, the maximum length of the vascular calcifications, and the maximum density of the calcifications for the 10-year pooled age categories rose with increasing age $(\mathrm{P}<0.001)$.

We reported mean and standard deviation (SD) for all continuous variables. For categoric variables, frequency distributions and percentages were stated. SPSS software (version 25.0) was used to perform the statistical analysis of this study. Logistic regression analysis was used to determine the relationship of BAC to CAC while adjusting for the common risk factors of age, chronic diseases as reported on a questionnaire before the mammography screening. The comparison of variables between the groups was registered using the Chi-squared test (or Fisher's exact test for specific parameters) and independent samples $t$-test. The Pearson correlation test assessed the correlation between C-IMT and continuous clinical and laboratory parameters; however, the correlation between C-IMT and nominal variables was evaluated by the Point-Biserial correlation analysis. Logistic regression analysis was performed by including the parameters which were significantly different between the two groups to determine independent predictors of BAC. Standardized $\beta$-regression coefficients and their significance from multiple linear regression analysis were recorded. A two-tailed $\mathrm{P}<0.005$ was reported as a statistically significant value.

\section{Results}

The patient population was diverse by age distribution and 120 BAC (+), 60 BAC (-) cases; overall, 180 patients were 
Table 2 Distribution of $\mathrm{BAC}(+)$ participants by chronic diseases

\begin{tabular}{lccc}
\hline Diseases & Subcategory & $\mathrm{N}$ & $\%$ \\
\hline $\mathrm{HT}$ & $(+)$ & 38 & 31.7 \\
& $(-)$ & 66 & 55.0 \\
$\mathrm{HM}$ & Unsure & 16 & 13.3 \\
& $(+)$ & 20 & 16.7 \\
& $(-)$ & 92 & 76.7 \\
Smoker & Unsure & 8 & 6.7 \\
& Current & 21 & 17.5 \\
& Past & 33 & 27.5 \\
Hyperlipidemia & Passive & 41 & 34.2 \\
& $(+)$ & 11 & 9.2 \\
& $(-)$ & 88 & 73.3 \\
& Unsure & 21 & 17.5 \\
\hline
\end{tabular}

BAC, breast arterial calcification; HT, hypertension; DM, diabetes mellitus.

included (Table 1). The prevalence of BAC was $8.4 \%$ (302 of 3,600 patients) in this study, consistent with the several previous studies $8-16 \%(17-19)$.

BAC (+) patients by concerning smoking history; 21 $(17.5 \%)$ of the patients participating in the study defined that they were currently smoking, 41 (34.2\%) women had a history of passive smoking exposure, and 33 (27.5\%) respondents had smoked in the past. Regarding HT, 38 (31.7\%) participants knew they had HT, $66(55.0 \%)$ respondents were not aware of HT, and $16(13.3 \%)$ patients were not sure whether they had high blood pressure. Regarding DM, 20 (16.7\%) patients had a story of DM, 92 (76.7\%) participants declared they did not have DM, and $8(6.7 \%)$ individuals were unsure. In BAC (+) study group concerning CAD, 10 (8.3\%) patients indicated that they had CAD, 74 (61.7\%) individuals stated that they did not have a history of CAD, and $36(30.0 \%)$ participants were unsure whether they had CAD. Regarding hyperlipidemia, 11 (9.2\%) participants had a clinical story of hyperlipidemia, $88(73.3 \%)$ females declared they had no hyperlipidemia, and $21(17.5 \%)$ patients were unsure. The mentioned data are presented in Table 2. In univariate analysis, age, hyperlipidemia, DM, HT, and smoking history were separate risk factors that significantly affected BAC development. However, only the impacts of age and DM were maintained in the logistic regression analysis $(\mathrm{P}<0.005)$,
Table 3 Mean C-IMT of participants by 10-year pooled age groups: the table displays the effects of age on C-IMT and BAC (C-IMT as a gold standard indicator of atherosclerosis)

\begin{tabular}{lccc}
\hline \multirow{2}{*}{ Age (years) } & \multicolumn{2}{c}{ Mean C-IMT $(\mathrm{mm})$} & \multirow{2}{*}{ P value } \\
\cline { 2 - 3 } & BAC $(-)$ & BAC $(+)$ & \\
\hline $40-49$ & 0.86 & 0.89 & 0.013 \\
$50-59$ & 0.92 & 1.03 & $<0.005$ \\
$60-69$ & 1.05 & 1.22 & $<0.001$ \\
$70-79$ & 1.23 & 1.35 & $<0.005$ \\
$\geq 80$ & 1.33 & 1.41 & 0.01 \\
Overall & 1.03 & 1,15 & $<0.005$ \\
\hline
\end{tabular}

C-IMT, carotid artery intima-media thickness; BAC, breast arterial calcification.

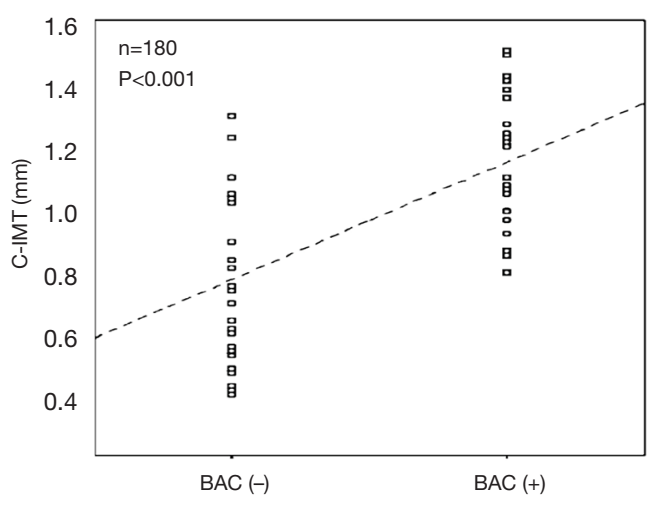

Figure 2 Graph demonstrating increased common carotid artery intima-media thickness (C-IMT) in BAC (+) group participants $(\mathrm{P}<0.005)$. The dashed line represents the significant correlation between the C-IMT and the presence of BAC on mammography (Point-Biserial correlation coefficient $\left(\mathrm{P}<0.005\right.$ and $\left.\mathrm{r}_{\mathrm{pb}}=0.6\right)$. There is a significant difference in mean C-IMT values between the two groups, statistically. BAC, breast arterial calcification.

while the significant effect of the other chronic diseases was distinctly disappeared $(\mathrm{P}>0.02)$.

Besides, carotid artery IMT measurements were recorded from the digital database for all 120 individuals participating in the study. We defined a significant relationship between age and C-IMT, and IMT increased as age advanced. A correlation was also found between the BAC score and C-IMT, as displayed in Table 3. The correlation was defined between IMT and BAC in the whole study group, as shown in Table 3 and Figure $2(\mathrm{P}<0.005)$.

BAC has been correlated with Agatston coronary calcium 
Table 4 Relationship between BAC \& Agatston Scores according to the quantitative evaluation

\begin{tabular}{|c|c|c|c|c|c|c|c|}
\hline BAC score & \multicolumn{6}{|c|}{ Agatston score, n (\%) } & $P$ value \\
\hline 0 & $35(19.4)$ & $9(5.0)$ & $8(4.4)$ & $5(2.8)$ & $3(1.7)$ & $60(33.3)$ & $<0.001$ \\
\hline $1-3$ & $15(8.3)$ & $26(14.4)$ & $17(9.4)$ & $16(8.9)$ & $12(6.7)$ & $86(47.8)$ & $<0.001$ \\
\hline $4-12$ & $1(0.6)$ & $8(4.4)$ & $11(6.1)$ & $8(4.4)$ & $6(3.3)$ & 34 (18.9) & $<0.001$ \\
\hline
\end{tabular}

BAC, breast arterial calcification.

Table 5 Relationship between BAC \& CAC scores: quantitative analysis of coronary atherosclerosis in the whole study group

\begin{tabular}{|c|c|c|c|c|c|}
\hline BAC score & \multicolumn{4}{|c|}{ CAC score } & $P$ value \\
\hline 0 (none) & $42(23.3)$ & $12(6.7)$ & $6(3.3)$ & $60(33.3)$ & $<0.001$ \\
\hline 1-3 (mild) & $33(18.3)$ & $36(20.0)$ & $17(9.4)$ & $86(47.8)$ & $<0.001$ \\
\hline 4-12 (moderate \& severe) & $7(3.9)$ & $15(8.3)$ & $12(6.7)$ & $34(18.9)$ & $<0.001$ \\
\hline
\end{tabular}

BAC, breast arterial calcification; CAC, coronary arterial calcification.

Table 6 Marginal frequency distributions to BAC \& CAC scores in the whole study group

\begin{tabular}{lcc}
\hline Score & $\mathrm{N}(\%)$ & P value \\
\hline CAC score: 0 & $82(45.6)$ & $<0.001$ \\
CAC score: $1-3$ & $63(35)$ & $<0.001$ \\
CAC score: $4-12$ & $35(19.4)$ & $<0.001$ \\
BAC score: 0 & $60(33.3)$ & $<0.001$ \\
BAC score: $1-3$ & $86(47.8)$ & $<0.001$ \\
BAC score: $4-12$ & $34(18.9)$ & $<0.001$ \\
\hline
\end{tabular}

BAC, breast arterial calcification; CAC, coronary arterial calcification.

score equal to or greater than $11(\mathrm{P}<0.001)$, and CAC score equal to or greater than 4 corresponds to a greater risk of developing CAD, as exposed in Tables 4-6. Agatston scores higher than 11 were established in $71.7 \%$ (86 of 120) of patients with BAC, as shown in Table 4. Women with BAC score 0; have Agatston scores of 0,1-10, 11-100, 101-400, and $401-1,000 ; 19.4 \%, 5.0 \%, 4.4 \%, 2.8 \%$, and $1.7 \%$, respectively. Patients with BAC score 1-3; have Agatston scores of $0,1-10,11-100,101-400$, and 401-1,000; $8.3 \%$, $14.4 \%, 9.4 \%, 8.9 \%$, and $6.7 \%$, respectively. Participants with BAC score 4-12; have Agatston scores of 0, 1-10, $11-100,101-400$, and $401-1,000 ; 0.6 \%, 4.4 \%, 6.1 \%, 4.4 \%$, and $3.3 \%$, respectively, as displayed in Table 4. On the other side, $26.7 \%$ (16 of 60 ) of patients without BAC had calcium scores equal to or greater than 11 . As a result, women with BAC score 0 ; have CAC scores of $0,1-3$, and $4-12 ; 23.3 \%$, $6.7 \%$, and $3.3 \%$, respectively. Patients with BAC score $1-3$; have CAC scores of $0,1-3$, and $4-12 ; 18.3 \%, 20.0 \%$, and 9.4\%, respectively. Participants with BAC score 4-12; have CAC scores of $0,1-3$, and $4-12 ; 3.9 \%, 8.3 \%$, and $6.7 \%$, respectively, as shown in Table 5 . In the whole study group, marginal frequency distributions according to BAC and CAC scores were presented in Table 6.

In the evaluation made by reference to the MESA study, that means when gender and age factors are considered, the presence of BAC showed a statistically significant correlation with percentile scores greater than 25 . As a result, it was observed that $68.3 \%(82 / 120)$ of patients with BAC had a percentile score greater than 25 , indicating the risk for CAD. A statistically significant relationship was determined between $\mathrm{BAC}(\mathrm{P}<0.001)$ and diabetes $(\mathrm{P}<0.005)$ as well. There was no association between BAC and hyperlipidemia $(\mathrm{P}>0.01)$. Consequently, according to the univariable and multivariable statistical analysis age, and diabetes were all found to be related to BAC. At the 
Table 7 Univariable and multivariable analyses in the BAC (+) study group to determine the impact of regarding factors on BAC (statistically significant if $\mathrm{P}$ value $\leq 0.005)$

\begin{tabular}{|c|c|c|c|c|c|c|}
\hline Diseases & \multicolumn{3}{|c|}{ Univariable } & \multicolumn{3}{|c|}{ Multivariable } \\
\hline HT & 1.31 & $1.06-1.74$ & 0.08 & 1.06 & $0.75-1.71$ & 0.45 \\
\hline Hyperlipidemia & 1.29 & $1.03-1.73$ & 0.08 & 0.93 & $0.62-1.48$ & 0.38 \\
\hline DM & 2.47 & $1.71-4.01$ & $<0.005$ & 2.08 & $1.59-3.35$ & $<0.005$ \\
\hline Age (10 years) & 3.36 & $2.75-4.15$ & $<0.001$ & 2.91 & $2.27-3.74$ & $<0.001$ \\
\hline
\end{tabular}

$\mathrm{OR}$, odds ratio; $\mathrm{Cl}$, confidence interval. BAC, breast arterial calcification; HT, hypertension; DM, diabetes mellitus.

Table 8 Correlation of BAC and CAC scores, results from advanced statistical analysis according to the 10-year age pool distribution

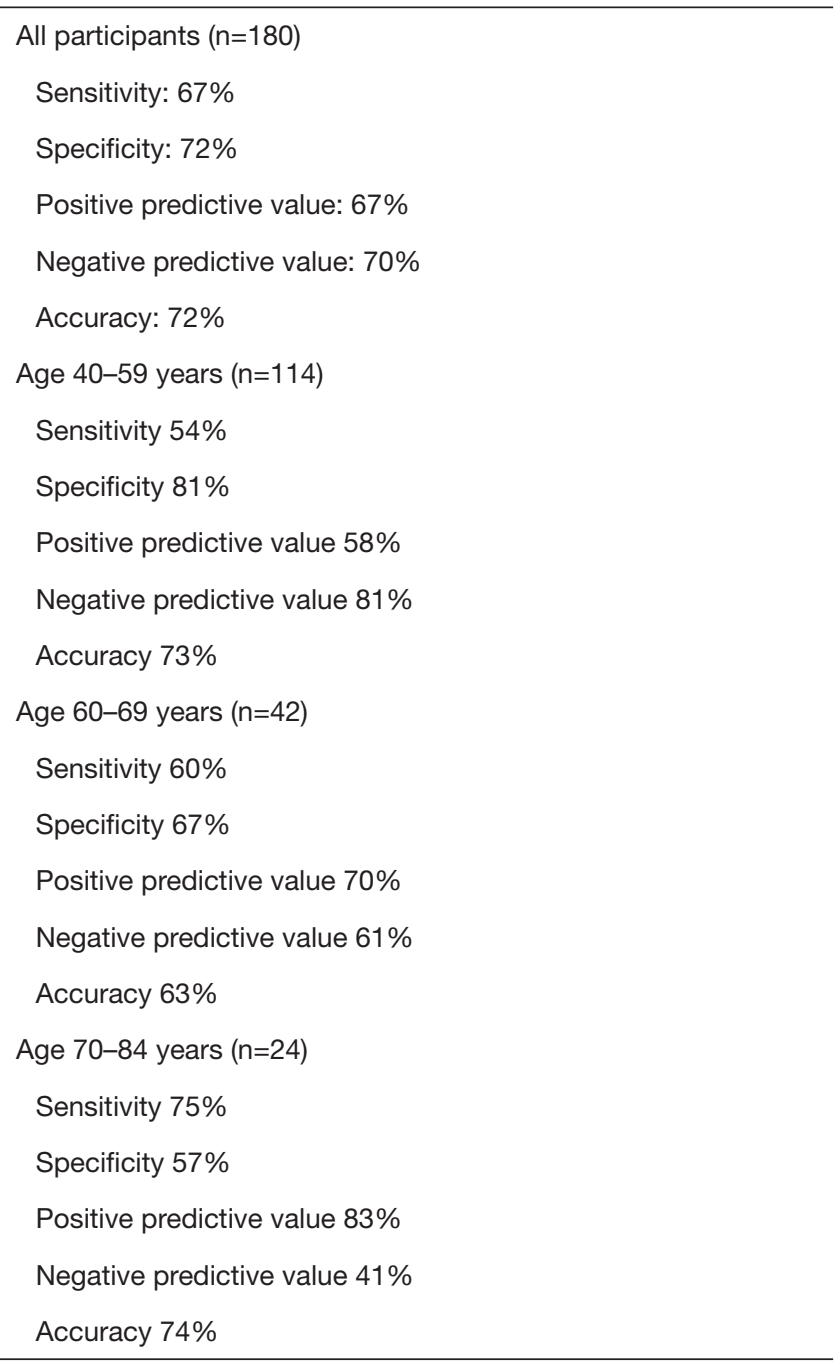

BAC, breast arterial calcification; CAC, coronary arterial calcification.

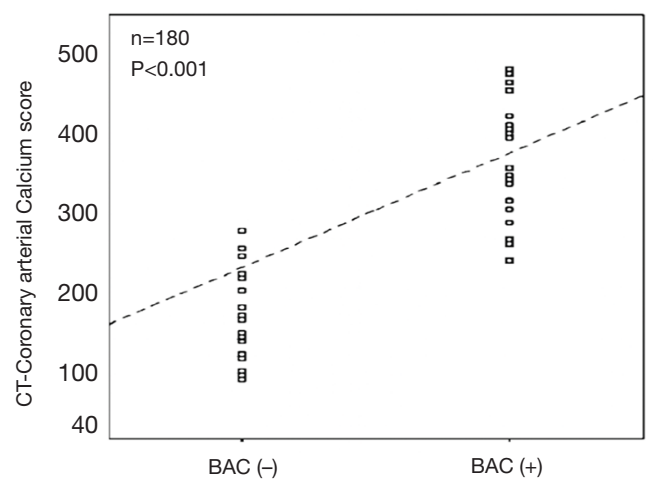

Figure 3 Graph demonstrating significantly increased CTcoronary artery calcium score (as Agatston Score) in BAC (+) group patients $(\mathrm{P}<0.001)$ when compared with BAC $(-)$ subjects. Maximum and minimum scores were excluded from reaching a more reliable report; meanwhile, the Point-Biserial correlation for statistical evaluation $\left(\mathrm{r}_{\mathrm{pb}}=0.5\right)$. BAC, breast arterial calcification.

same time, there was no association between HT and hyperlipidemia with BAC (Table 7).

The sensitivity, specificity, positive predictive value, negative predictive value, and accuracy of BAC $>0$ for the presence of $\mathrm{CAC}>0$ was $67 \%, 72 \%, 67 \%, 70 \%$, and $72 \%$, respectively, as shown in Table 8 . With increasing age, sensitivity has increased from $54 \%$ to $75 \%$, and specificity has decreased from $81 \%$ to $57 \%$. Similarly, the positive predictive value increased from $58 \%$ to $83 \%$, and the negative predictive value decreased from $81 \%$ to $41 \%$ with increasing age (Table 8). The associations of the BAC score and the CAC score were all highly significant $(\mathrm{P}<0.001)$.

The agreement between BAC and CAC scores was also highly significant, as shown in Figure $3(\mathrm{P}<0.001)$.

Case examples demonstrate that, in the BAC (-) case 

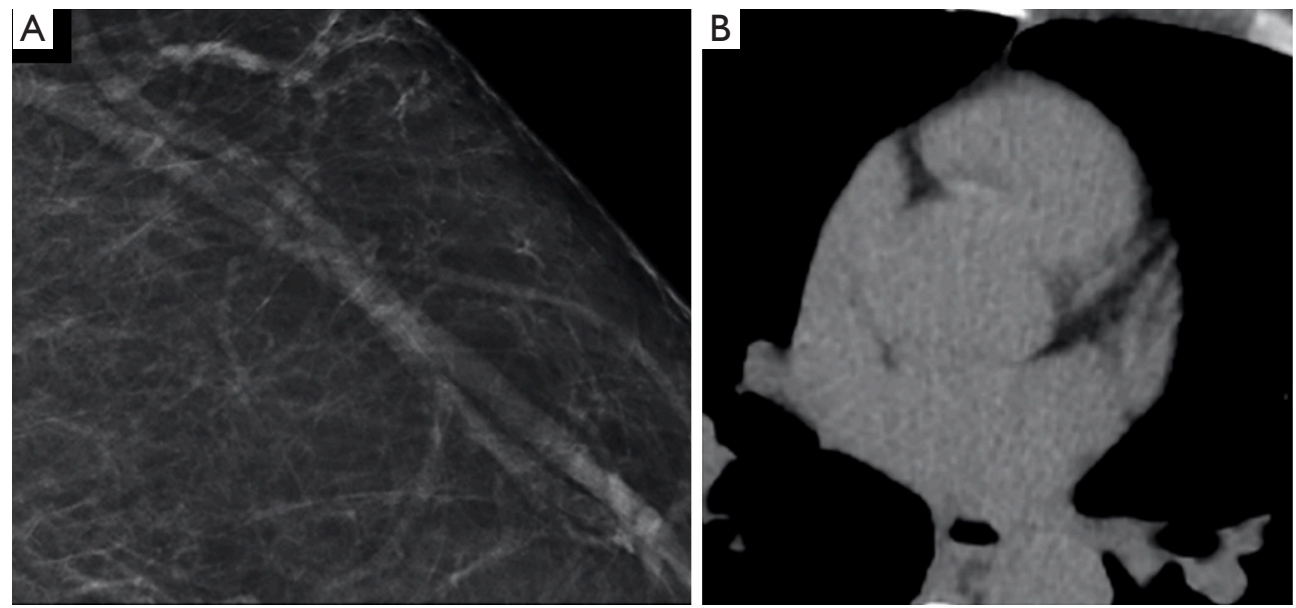

Figure 4 Mammography image (A) of a 46-year-old female displays a BAC score of 0, and coronary calcium scoring CT image (B) of the same participant exhibits both Agatston and CAC scores of 0 . BAC, breast arterial calcification; CAC, coronary arterial calcification.
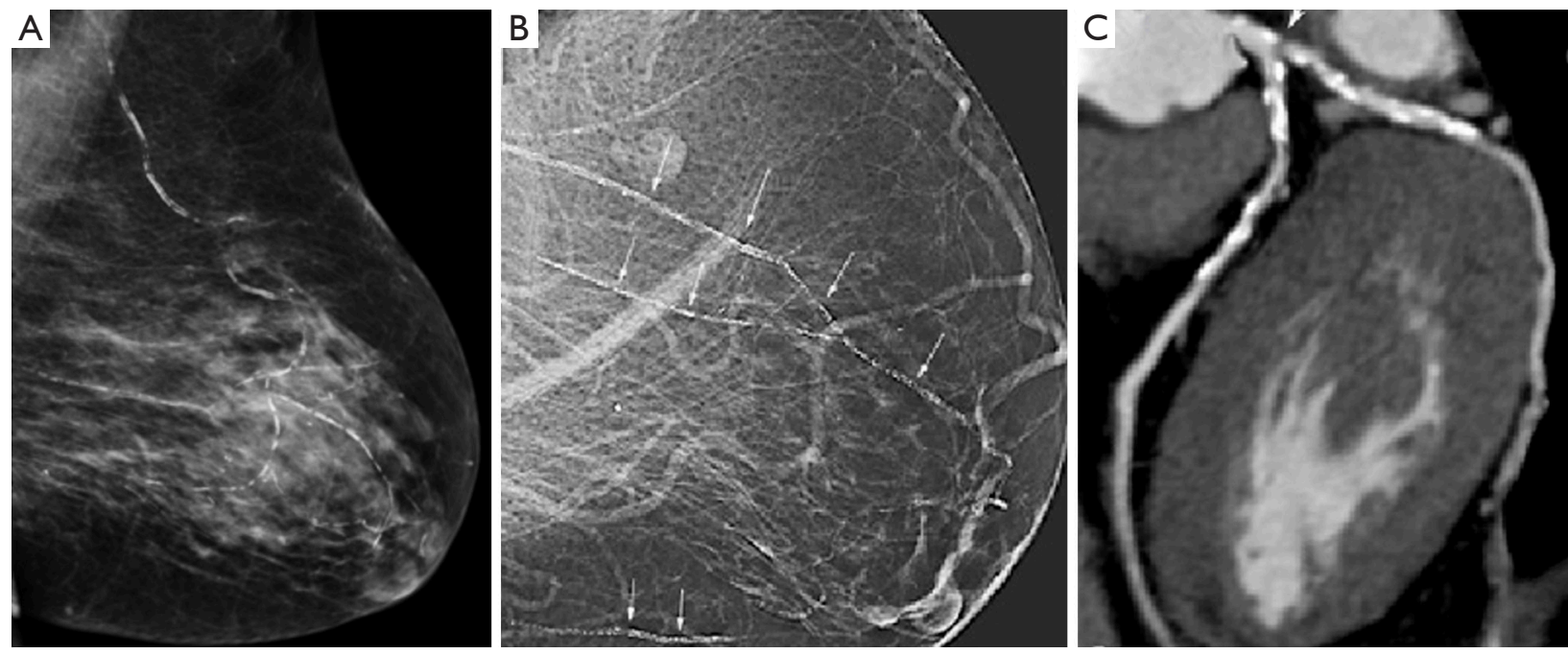

Figure 5 Mammography image (A) belongs to a 73-year-old female with a BAC score of 11 . Another mammography image (B) displays a 74-year-old female with a BAC score of 9, and coronary CT angiography (C) of the same patient confirms a CAC score of 8 and an Agatston score of 467. CT, computed tomography; BAC, breast arterial calcification; CAC, coronary arterial calcification.

group, mammography and coronary calcium scoring CT images of the individual with Agatston and CAC scores of 0 are shown in Figure 4. In the BAC (+) case group, mammography and CT images of 73 and 74-year-old patients with signs of coronary atherosclerosis, BAC score 9, Agatston score 467, and CAC score 8, are depicted in Figure 5. In addition, mammography and CT images of a 67-year-old individual with coronary atherosclerosis findings, BAC score of 5, Agatston score of 32, and CAC score of 3, are demonstrated in Figure 6. Mammography and CT images of a 75-year-old woman with prominent signs of coronary atherosclerosis with a BAC score of 8 , Agatston score of 254, and CAC score of 7 are displayed in Figure 7. These samples demonstrate the correlation between the BAC score and the Agatston and CAC scores.

\section{Discussion}

In the United States, approximately 250,000 women die of ASCVD annually. More than $60 \%$ of these women had 

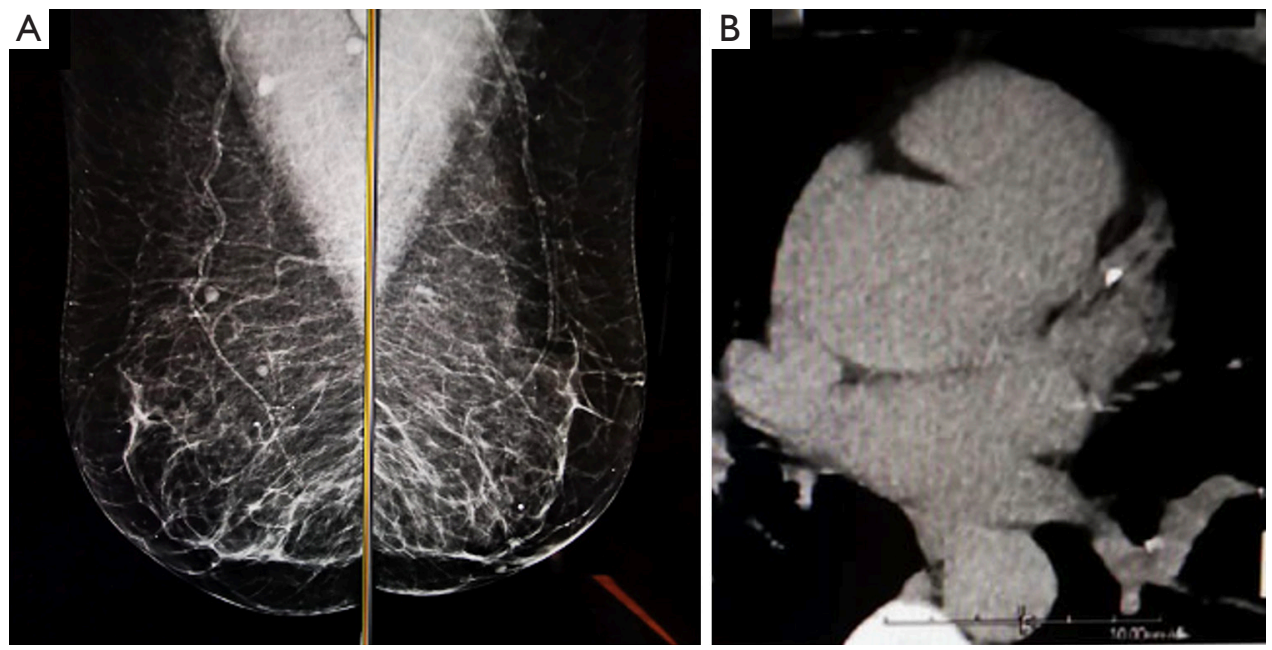

Figure 6 Bilateral mediolateral oblique mammography images (A) of a 67-year-old participant show a BAC score of 5, and coronary calcium scoring CT image (B) of the same patient depicts a CAC score of 3, Agatston score 32. CT, computed tomography; BAC, breast arterial calcification; CAC, coronary arterial calcification.
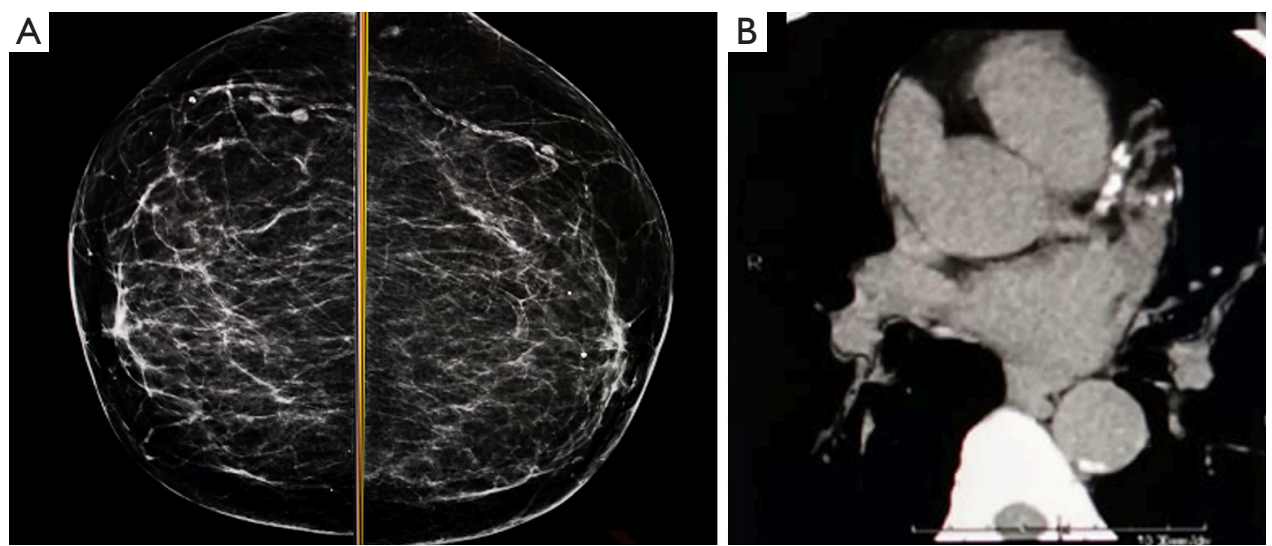

Figure 7 Bilateral craniocaudal mammography images (A) demonstrate a 75-year-old respondent with a BAC score of 8 , and coronary calcium scoring CT image (B) of the same patient represents a CAC score of 7, Agatston score of 254. CT, computed tomography; BAC, breast arterial calcification; CAC, coronary arterial calcification.

no initial symptoms, demonstrating the importance of cardiovascular risk assessment and early diagnosis of CAD. Imaging modalities, such as CT that detects coronary artery calcifications, plain radiographs that define aortic calcifications, and mammography to identify BAC, may assist in predicting cardiovascular risk. There are conflicting outcomes in the literature regarding the clinical significance of breast vascular calcifications reported in mammography. Some authors claim that BAC can be helpful in determining CAD risk, while some others suggest that it is not $(12,14,21)$.

We aimed to reveal the correlation of BAC with age and
CAC. Moreover, the study investigated the relationship between BAC and chronic diseases such as DM, hyperlipidemia, HT, and smoking. As previously reported in several studies, age is the most critical factor influencing the prevalence of BAC. It has been proved that BAC increases with aging, as expected $(22,23)$. Pre-existing CAD is also correlated with a higher prevalence of BAC (24). The mean age of the study group is $56.2( \pm 7.1 \mathrm{SD})$, as occurred that the rate is consistent with the current literature data. However, the average age of 60 and above, or a higher rate of participants over 60 years of age, would increase the 
value and reliability of correlation studies. In this study, approximately $1 / 3$ of the cases were 60 years of age or older. Data indicates similar characteristics regarding the age distribution between some other studies in the literature and this study (25).

In the literature so far, the prevalence of BAC on mammography has been reported at very different frequencies (21). The prevalence of BAC varies from $1 \%$ to $49 \%$ in previous studies $(21-28)$. Such a significant difference in prevalence may be explained by variations in sensitivity of mammography equipment and the heterogeneity of the study groups (16). Our study detected BAC in $8.4 \%$ of the participants who underwent mammography within 5 years.

BAC is a mammographic finding that is not associated with cancer (29). That is reported as a benign finding in mammographic evaluation. The positive correlation between age and BAC is emphasized in the literature, and there is no conflicting sentence about that $(12,26,28-30)$. BAC is encountered as an incidental mammographic finding that represents degenerative calcific changes of the breast arteries. These calcific changes occur on the intimal or medial layers of mammary arteries, most likely by increasing age. These different locations represent discrete pathophysiological processes $(9,3132)$. Intimal calcification is an active process similar to bone formation and occurs by expressing growth factors, matrix proteins, and other bonerelated proteins (33). The process is related to inflammatory cells, lipid deposits, and vascular smooth muscle cells. Contrarily, medial arterial (Mönckeberg-type) calcification has been described as secondary to aging, diabetes, endstage renal disease, neuropathy, and several rare genetic syndromes (16,34-37). Research on medial calcification showed that it is associated with type II diabetes and increased CVD mortality. And some others have confirmed that medial calcification is an independent predictor of CVD events in patients with diabetes or end-stage kidney disease (17). In this study, consistent with the literature, we have stated that aging increases the prevalence of BAC.

By the advantage of relatively low cost and easy accessibility of mammogram screening; possible association between BAC, CVD, stroke, carotid artery disease, and peripheric arterial disease, the study of BAC and its relation to these may provide clinical benefit for diagnosis and prevention of these comorbid situations (38). It has been suggested that BAC must be reported as part of routine mammography examinations. Patients, radiologists, and referring physicians can use the information as part of collective decision-making regarding cardiac evaluation to maximize the benefit of preventive cardiology strategies $(11,17,39-42)$. Besides, mammography is routinely used as a screening test for breast cancer. Its use to detect BAC will not charge any extra cost or radiation exposure.

Smoking, HT, and hyperlipidemia are known and proven risk factors for atherosclerosis $(11,31,32,42)$. Our study determined that these chronic diseases did not significantly affect the BAC score, according to the quantitative statistical multivariate analysis. Such a result is related to BAC emerging secondary to the medial calcific type of atherosclerotic process. Unexpectedly, some researchers have reported a lower incidence of BAC in women who smoke compared to nonsmoking women $(12,18,24)$. In this study, we have defined no significant relationship between BAC and smoking. In the literature, there are conflicting conclusions about the relationship of BAC with DM, HT, and hyperlipidemia. While some researchers reported a significant relationship between BAC and DM $(12,17,24,32)$, HT $(17,32,43,44)$, and hyperlipidemia (29); some others found no significant relationship between BAC and DM $(11,25)$, HT $(11,25)$, or hyperlipidemia (25). In our study, we did not find any relationship between BAC and HT or hyperlipidemia. Differently, we detected a significant statistical relationship between BAC and DM. Everhart et al. also described a correlation between DM and atherosclerosis, medial arterial calcification (14).

Some researchers described that BAC was predictive of subsequent CACs (25). Dale et al. stated that in the mammographic examination, women with myocardial infarction (MI) history had significantly higher frequencies of BAC than women without a history of MI. (12). Besides, another study determined an association between mammographically detected arterial calcifications and ASCVD (33). Otherwise, to the conclusions of their research, Ak et al. expressed that BAC did not help determine coronary atherosclerosis (45). Our study found that $\mathrm{CAD}$ is one of the critical risk factors of $\mathrm{BAC}$ and higher BAC scores correlate with higher CAC scores. Statistically significant results were obtained in both univariate and regression analyses.

We assume that some researchers failed to detect a significant relationship between BAC and CAD $(44,46)$, maybe because they did not grade vascular calcification in the breast, mainly by not scoring BAC. A low BAC score reveals a lower risk for $\mathrm{CAD}$, according to the quantitative evaluation. However, it is inaccurate to expect the BAC to be a correct marker of CAD only by identifying that as 
positive or negative. If both parameters are considered and graded quantitatively as in this study, more rigorous and detailed research can correlate relevant data. A separate evaluation for the statistical significance of the CAC score and Agatston score with BAC also contributed to this result. Thus, a new and easily accessible screening and early diagnosis procedure may occur. Such protocol to image the breasts for cancer screening and the heart for coronary atherosclerosis at the same session could represent an exciting dual-screening approach. Then this procedure may accurately be an indicator of mild or severe ASCVD.

There are a few limitations in our study, which should be considered. Ethnic origin, an essential factor for atherosclerosis, could not be a subject of this study since all patients in the study group belonged to the same race. Whether there is a significant difference between different ethnic groups could not be investigated in this study. Besides, some researchers reported an essential relationship between BAC and alcohol consumption (19). However, secondary to the inadequate anamnesis, alcohol consumption and the BAC relationship could not be researched in this study. While determining the BAC (-) group in our study, we selected the age distribution similar to the patient group. On the other hand, we could not access detailed clinical and laboratory data for some of the patients in this group; we set up the group randomly without considering the chronic disease histories. Also, univariate and multivariate statistical analyzes were conducted without grading the smoking history as packyears. This is because the factor in question is not the main subject of the study. End-stage CKD is a significant risk factor of atherosclerosis $(1,24,40)$; however, it is not included among chronic diseases in this study since it was set up as a separate study subject. Moreover, the fact that body mass index was not considered and examined in our study may be a disadvantage.

In conclusion, according to the outcomes of the present multi-modality study BAC is strongly associated with aging and high BAC scores are correlated with higher CAC score, that means moderate or severe BAC may be related with CAD. Consequently, we suggest that the BAC scoring system can be used as an indicator of determining the presence of CVD and higher cardiovascular risk, particularly in women younger than 60 years old. BAC does predict moderate and increased coronary artery calcium scores, which indicates a higher risk for coronary atherosclerosis. Furthermore, a statistically significant correlation exists between BAC and cardiac risk factors such as diabetes. Our study suggests that BAC described on mammography screening can indicate an increased risk of developing $\mathrm{CAD}$, and early diagnosis can prevent morbidity and mortality related to that.

\section{Acknowledgments}

Funding: None.

\section{Footnote}

Conflicts of Interest: Both authors have completed the ICMJE uniform disclosure form (available at https://dx.doi. org/10.21037/qims-21-98). The authors have no conflicts of interest to declare.

Ethical Statement: The authors are accountable for all aspects of the work in ensuring that questions related to the accuracy or integrity of any part of the work are appropriately investigated and resolved. The study was conducted in accordance with the Declaration of Helsinki (as revised in 2013). The study was approved by institutional ethics committee of Başkent University Faculty of Medicine and informed consent was taken from all the patients.

Open Access Statement: This is an Open Access article distributed in accordance with the Creative Commons Attribution-NonCommercial-NoDerivs 4.0 International License (CC BY-NC-ND 4.0), which permits the noncommercial replication and distribution of the article with the strict proviso that no changes or edits are made and the original work is properly cited (including links to both the formal publication through the relevant DOI and the license). See: https://creativecommons.org/licenses/by-nc-nd/4.0/.

\section{References}

1. Nasir K, McEvoy JW. Recognizing Breast Arterial Calcification as Atherosclerotic CVD Risk Equivalent: From Evidence to Action. JACC Cardiovasc Imaging 2016;9:361-3.

2. Amin NP, Martin SS, Blaha MJ, Nasir K, Blumenthal RS, Michos ED. Headed in the right direction but at risk for miscalculation: a critical appraisal of the 2013 ACC/AHA risk assessment guidelines. J Am Coll Cardiol 2014;63:2789-94.

3. Bild DE, Folsom AR, Lowe LP, Sidney S, Kiefe C, Westfall AO, Zheng ZJ, Rumberger J. Prevalence and 
correlates of coronary calcification in black and white young adults: the Coronary Artery Risk Development in Young Adults (CARDIA) Study. Arterioscler Thromb Vasc Biol 2001;21:852-7.

4. Budoff MJ, Nasir K, Mao S, Tseng PH, Chau A, Liu ST, Flores F, Blumenthal RS. Ethnic differences of the presence and severity of coronary atherosclerosis. Atherosclerosis 2006;187:343-50.

5. Wayhs R, Zelinger A, Raggi P. High coronary artery calcium scores pose an extremely elevated risk for hard events. J Am Coll Cardiol 2002;39:225-30.

6. Arad Y, Spadaro LA, Goodman K, Newstein D, Guerci AD. Prediction of coronary events with electron beam computed tomography. J Am Coll Cardiol 2000;36:1253-60.

7. Hofmann Bowman MA, McNally EM. Genetic pathways of vascular calcification. Trends Cardiovasc Med 2012;22:93-8.

8. Micheletti RG, Fishbein GA, Currier JS, Fishbein MC. Mönckeberg sclerosis revisited: a clarification of the histologic definition of Mönckeberg sclerosis. Arch Pathol Lab Med 2008;132:43-7.

9. Chen NX, Moe SM. Arterial calcification in diabetes. Curr Diab Rep 2003;3:28-32.

10. Margolies L, Salvatore M, Hecht HS, Kotkin S, Yip R, Baber U, Bishay V, Narula J, Yankelevitz D, Henschke C. Digital Mammography and Screening for Coronary Artery Disease. JACC Cardiovasc Imaging 2016;9:350-60.

11. Suh JW, Yun B. Breast Arterial Calcification: A Potential Surrogate Marker for Cardiovascular Disease. J Cardiovasc Imaging 2018;26:125-34.

12. Maas AH, van der Schouw Y'T, Beijerinck D, Deurenberg JJ, Mali WP, van der Graaf Y. Arterial calcifications seen on mammograms: cardiovascular risk factors, pregnancy, and lactation. Radiology 2006;240:33-8.

13. Crystal P, Zelingher J, Crystal E. Breast arterial calcifications as a cardiovascular risk marker in women. Expert Rev Cardiovasc Ther 2004;2:753-60.

14. Baum JK, Comstock CH, Joseph L. Intramammary arterial calcifications associated with diabetes. Radiology 1980;136:61-2.

15. Leinster SJ, Whitehouse GH. Factors which influence the occurrence of vascular calcification in the breast. Br J Radiol 1987;60:457-8.

16. Tomson C. Vascular calcification in chronic renal failure. Nephron Clin Pract 2003;93:c124-30.

17. London GM, Guérin AP, Marchais SJ, Métivier F, Pannier B, Adda H. Arterial media calcification in end-stage renal disease: impact on all-cause and cardiovascular mortality.
Nephrol Dial Transplant 2003;18:1731-40.

18. Shemesh J, Henschke CI, Shaham D, Yip R, Farooqi AO, Cham MD, McCauley DI, Chen M, Smith JP, Libby DM, Pasmantier MW, Yankelevitz DF. Ordinal scoring of coronary artery calcifications on low-dose CT scans of the chest is predictive of death from cardiovascular disease. Radiology 2010;257:541-8.

19. Kim H, Greenberg JS, Javitt MC. Breast calcifications due to Mönckeberg medial calcific sclerosis. Radiographics 1999;19:1401-3.

20. Everhart JE, Pettitt DJ, Knowler WC, Rose FA, Bennett PH. Medial arterial calcification and its association with mortality and complications of diabetes. Diabetologia 1988;31:16-23.

21. Bui QM, Daniels LB. A Review of the Role of Breast Arterial Calcification for Cardiovascular Risk Stratification in Women. Circulation 2019;139:1094-101.

22. Kataoka M, Warren R, Luben R, Camus J, Denton E, Sala E, Day N, Khaw KT. How predictive is breast arterial calcification of cardiovascular disease and risk factors when found at screening mammography? AJR Am J Roentgenol 2006;187:73-80.

23. Moshyedi AC, Puthawala AH, Kurland RJ, O'Leary DH. Breast arterial calcification: association with coronary artery disease. Work in progress. Radiology 1995;194:181-3.

24. Dale PS, Mascarhenas C, Richards M, Mackie G. Mammography as a screening tool for coronary artery disease. J Surg Res 2008;148:1-6.

25. Chadashvili T, Litmanovich D, Hall F, Slanetz PJ. Do breast arterial calcifications on mammography predict elevated risk of coronary artery disease? Eur J Radiol 2016;85:1121-4.

26. Taşkin F, Akdilli A, Karaman C, Unsal A, Köseoğlu K, Ergin F. Mammographically detected breast arterial calcifications: indicators for arteriosclerotic diseases? Eur J Radiol 2006;60:250-5.

27. Rotter MA, Schnatz PF, Currier AA Jr, O'Sullivan DM. Breast arterial calcifications (BACs) found on screening mammography and their association with cardiovascular disease. Menopause 2008;15:276-81.

28. Akinola RA, Ogbera OA, Onakoya JA, Enabulele CE, Fadeyibi IO. Mammograms and breast arterial calcifications: looking beyond breast cancer: a preliminary report. BMC Res Notes 2011;4:207.

29. Iribarren C, Go AS, Tolstykh I, Sidney S, Johnston SC, Spring DB. Breast vascular calcification and risk of coronary heart disease, stroke, and heart failure. J Womens 
Health (Larchmt) 2004;13:381-9.

30. Loberant N, Salamon V, Carmi N, Chernihovsky A. Prevalence and Degree of Breast Arterial Calcifications on Mammography: A Cross-sectional Analysis. J Clin Imaging Sci 2013;3:36.

31. Shanahan CM, Proudfoot D, Tyson KL, Cary NR, Edmonds M, Weissberg PL. Expression of mineralisationregulating proteins in association with human vascular calcification. Z Kardiol 2000;89 Suppl 2:63-8.

32. Wallin R, Wajih N, Greenwood GT, Sane DC. Arterial calcification: a review of mechanisms, animal models, and the prospects for therapy. Med Res Rev 2001;21:274-301.

33. Trion A, van der Laarse A. Vascular smooth muscle cells and calcification in atherosclerosis. Am Heart J 2004;147:808-14.

34. Mönckeberg JG. Über die reine Mediaverkalkung der Extremitätenarterien und ihr Verhalten zur Arteriosklerose. Virchows Arch Pathol Anat Physiol Klin Med 1903;171:141-67.

35. Edmonds ME, Morrison N, Laws JW, Watkins PJ. Medial arterial calcification and diabetic neuropathy. Br Med J (Clin Res Ed) 1982;284:928-30.

36. Farzaneh-Far A, Proudfoot D, Shanahan C, Weissberg PL. Vascular and valvar calcification: recent advances. Heart 2001;85:13-7.

37. Shanahan CM, Cary NR, Salisbury JR, Proudfoot D, Weissberg PL, Edmonds ME. Medial localization of mineralization-regulating proteins in association with Mönckeberg's sclerosis: evidence for smooth muscle cell-mediated vascular calcification. Circulation 1999; 100:2168-76.

38. Shah N, Chainani V, Delafontaine P, Abdo A, Lafferty J, Abi Rafeh N. Mammographically detectable breast arterial calcification and atherosclerosis. Cardiol Rev 2014;22:69-78.

Cite this article as: Kadığlu A, Bahadır S. Breast arterial calcifications as an indicator of atherosclerotic cardiovascular disease: comparative analysis of coronary computed tomography scoring systems and carotid intima-media thickness. Quant Imaging Med Surg 2022;12(1):457-469. doi: 10.21037/qims-21-98
39. Mantas D, Markopoulos C. Screening mammography: usefulness beyond early detection of breast cancer. Atherosclerosis 2016;248:1.

40. Polonsky TS, Greenland P. Breast Arterial Calcification: Expanding the Reach of Cardiovascular Prevention. Circulation 2017;135:499-501.

41. Zuin M, Rigatelli G, Scaranello F, Ribecco SG, Picariello C, Zuliani G, Faggian G, Zonzin P, Roncon L. Breast arterial calcifications on mammography and coronary artery disease: A new screening tool for cardiovascular disease? Int J Cardiol 2016;220:310-1.

42. Yoon YE, Kim KM, Lee W, Han JS, Chun EJ, Ahn S, Choi SI, Yun B, Suh JW. Breast Arterial Calcification is Associated with the Progression of Coronary Atherosclerosis in Asymptomatic Women: A Preliminary Retrospective Cohort Study. Sci Rep 2020;10:2755.

43. Sedighi N, Radmard AR, Radmehr A, Hashemi P, Hajizadeh A, Taheri AP. Breast arterial calcification and risk of carotid atherosclerosis: focusing on the preferentially affected layer of the vessel wall. Eur J Radiol 2011;79:250-6.

44. Henkin Y, Abu-Ful A, Shai I, Crystal P. Lack of association between breast artery calcification seen on mammography and coronary artery disease on angiography. J Med Screen 2003;10:139-42.

45. Ak M, Sivrioğlu AK, Sönmez G, İncedayi M, Aribal S, Sağlam M, et al. Investigation of the risk of cardiovascular disease in patients with breast artery calcification. Anatol J. Clin Investig 2012;6:246-50.

46. Yalçin Şafak K, Eratalay A, Dülger İliş E, Umarusman Tanju N. The relationship of breast arterial calcification detected in mammographic examinations with cardiovascular diseases, cardiovascular risk factors, parity, and breastfeeding. Turk J Med Sci 2016;46:641-5. 